\title{
Surfactant - Where are we in 2003?
}

JF Lewis MD

$S^{u+s}$

urfactant research has progressed over the past several years $\checkmark$ to the extent that exogenous surfactant administration in patients with the acute respiratory distress syndrome (ARDS) is now being evaluated. Unfortunately, clinical responses have been variable, and we now need to take a look at how surfactant is altered in this disease so that more effective treatment strategies can be developed. This review briefly discusses the biophysical and host defense properties of surfactant, the impact of mechanical ventilation (MV) on the endogenous surfactant system and the most recent clinical data involving exogenous surfactant administration in patients with ARDS. Discussions regarding future directions of surfactant research both in ARDS and diseases other than acute lung injury are included.

\section{COMPOSITION AND METABOLISM OF ENDOGENOUS SURFACTANT}

\section{Composition}

Pulmonary surfactant is the inner lining layer of the lung synthesized in type II cells and secreted into the alveolar space. Once secreted from these cells, surfactant spreads to form a surface film over the alveolar space, which ultimately extends up into the airways. Bronchoalveolar lavage samples obtained from normal lungs and analyzed for surfactant composition reveal that most of the endogenous surfactant is lipid, with the most prominent lipid being disaturated phosphatidylcholine. Other lipids include phosphatidylglycerol and phosphatidylinositol, as well as some unsaturated phospholipids and cholesterol (1). There are four specific surfactant proteins associated with these lipids, and they are termed SP-A, - B, -C and -D. Two of these proteins (SP-B and $-\mathrm{C}$ ) are hydrophobic and are very closely associated with the lipids. Indeed, natural exogenous surfactant preparations used for neonates with RDS predominantly contain disaturated phosphatidylcholine and varying amounts of hydrophobic proteins. The two hydrophilic proteins (SP-A and -D), on the other hand, are excluded from these surfactant preparations during the purification procedure, and currently, no exogenous surfactant preparations exist that contain hydrophilic proteins.

\begin{abstract}
Metabolism
Once secreted from the type II cell, the intracellular surfactant storage granules known as lamellar bodies unravel to form organized, lattice-like structures called tubular myelin. These latter forms are direct precursors to the surfactant film at the air-liquid interface (2). With respiratory motion, components of the film are extruded back into the subphase in small vesicular forms, which are either taken up by the type II cell for recycling or cleared from the air space via alveolar macrophages. The tubular myelin-like forms are more dense structures, are the functionally active component of surfactant and are referred to as large aggregates (LAs). The smaller vesicular forms are functionally inactive and are called small aggregates (SAs). The formation of LAs, adsorbtion to the surface film and production of SAs are in constant metabolic sequence. Indeed, the conversion of LAs into SAs has been studied using both in vitro and in vivo techniques, and has been shown to be dependent on serine protease activity, as well a phasic change in the surface area of the surfactant film (3). It was the recognition of the importance of surface area change in LA conversion that led to a series of studies investigating the impact of MV on alveolar surfactant metabolism.
\end{abstract}

\section{THE EFFECTS OF MV ON SURFACTANT AGGREGATE CONVERSION}

Based on the observation that conversion of LAs into SAs is dependent on surface area changes, animal studies have evaluated the effects of using different tidal volumes during MV on LA conversion (4). These studies have shown that higher tidal volumes resulted in an increased conversion of LAs into SAs, which, in the setting of acute lung injury, resulted in progressive lung dysfunction. This lung dysfunction was due, in part, to changes in the functional pools of surfactant (ie, increased SA relative to LA pools). Ventilatory modes minimizing phasic changes in surface area, or 'low stretch' strategies, were superior, as was the novel mode of MV called high frequency oscillation. Interestingly, recent clinical trials have shown that low stretch MV strategies resulted in improved 
mortality rates in patients with ARDS, and animal studies showed that low tidal volume strategies preserved surfactant in the functional LA forms (4). These findings have provided some insight into the mechanisms responsible for improved outcomes of patients managed with low stretch ventilation strategies in clinical trials.

\section{THE FUNCTIONS OF PULMONARY SURFACTANT Biophysical function}

Classically, the main function of pulmonary surfactant is related to its ability to lower surface tension at the air-liquid interface. Physiologically, this can be explained by LaPlace's Law $(\Delta \mathrm{P}=2 \mathrm{~T} / \mathrm{r})$, whereby the relative change in pressure $(\Delta \mathrm{P})$ within an alveolus (or the tendency for an alveolus to collapse) is dependent on two times the surface tension $(\mathrm{T})$ divided by the radius ( $r$ ) of the alveolus. In this context, if surface tension was high and did not change, either due to a primary deficiency of surfactant or an alteration in surfactant function, alveoli would tend to collapse as they became smaller during exhalation. Pulmonary surfactant serves to maintain alveolar stability at low lung volumes by decreasing surface tension at the air-liquid interface, particularly on compression of the film during exhalation. This serves to decrease the work of breathing. Indeed, the administration of exogenous surfactant to preterm infants born deficient in this material has had a significant impact on infant mortality.

\section{Nonbiophysical function}

Surfactant has also been shown to impact ciliary function within the airways, enhance and optimize bacterial uptake and killing within alveolar macrophages, and influence mediator release from activated inflammatory cells (5). In vivo studies have also shown that transgenic animals deficient in SP-A were more susceptible to both bacterial and viral infections, because clearance of the organisms was impaired and a greater inflammatory response was observed in the knockout mice compared with wild types (6). Together, these studies suggest that surfactant has very important host defense functions in addition to its biophysical role. Clinically, these functions may have important implications in the initiation and development of acute lung injury before significant lung dysfunction is present.

\section{EXOGENOUS SURFACTANT THERAPY - RATIONALE AND CURRENT STATUS}

Currently, exogenous surfactant has only been tested in patients with established, severe ARDS. In this setting, patients are extremely hypoxemic and have decreased lung compliance and severe respiratory failure. Evaluation of surfactant abnormalities associated with severe lung injury have shown an increase in the poorly functioning SA forms relative to LAs, a decrease in the disaturated phosphatidylcholine concentration within the remaining LAs and a decrease in the SP levels (7). An impairment of surfactant function was also observed, mainly due to inhibition of the surfactant from the influx of serum proteins into the air space due to the lung injury. Current studies are also evaluating the potential for surfactant to be oxidized and/or nitrated due to the abundance of reactive oxygen species present in these airways (8). Based on these observations and several preclinical clinical studies evaluating exogenous surfactant in animal models of lung injury, administration of surfactant to patients with ARDS has been rationalized. Unfortunately, results of clinical trials conducted to date have been variable.

Two recent phase III clinical trials evaluating a recombinant SP-C-based exogenous surfactant called Venticute (ALTANA Pharma, Germany) showed no overall change in mortality compared with standard treatment (personal communication with ALTANA Pharma). Although these results were obviously disappointing, post hoc evaluation of these patients revealed that some subgroups did, in fact, exhibit significant improvements in response to the surfactant. For example, patients with 'direct' lung injuries, such as those induced by pneumonia and/or aspiration, had superior responses compared with those with systemic sepsis or trauma-induced ARDS. It was also recognized that most patients who filled the definition of ARDS had multiple etiologies resulting in respiratory failure, so that in some patients, there was a complex pathophysiological process ongoing that may have limited the efficacy of any therapy, let alone surfactant. These findings have led to the design of another prospective, randomized, clinical trial evaluating the role of Venticute in patients with 'direct' lung injuries; this study involves administering the surfactant relatively early (before $48 \mathrm{~h}$ ) after the onset of MV. This study is now underway in Europe and is due to commence shortly in North America.

Factors influencing the efficacy of exogenous surfactant A number of factors can influence a patient's response to exogenous surfactant administration (9). First, is the specific surfactant preparation used. In general, natural surfactant products such as bovine lipid extract surfactant (BLES Biochem, Canada), Infasurf (Forest Laboratories, USA), Alveofact (Boehringer Ingelheim, Germany) and Curosurf (Chiesi Pharmaceuticals, Italy) are all superior to synthetic products that contain no surfactant-associated proteins (ie, Exosurf [Glaxo Welcome, USA] and artificial lung-expanding compound [Britannia Pharmaceuticals, UK]). Newer recombinant products (ie, Venticute, which is based on a recombinant SP-C protein, and Surfaxin [Discovery Laboratories, USA], which is based on a SP-B-like peptide) appear to be comparable with the natural products, at least in preclinical testing.

The method used to deliver the surfactant can also influence the host's response to this therapy, and current techniques include instillation of liquid boluses directly into the lungs through the endotracheal tube, as well as administration of the surfactant via bronchoscopic lavage. The latter technique may facilitate serum protein removal from the alveolar space while permitting surfactant deposition. Currently, there is no role for aerosolization in severe ARDS due to the nonuniform nature of the injury in these patients. Finally, given the impact of these factors on efficacy, it would appear that the optimal dose of surfactant is dependent on the specific preparation used, as well as the method of delivery.

Based on current knowledge, therefore, patients with severe lung injury associated with proteinaceous pulmonary edema 
likely require administration of large quantities of a biophysically active surfactant to overcome protein inhibition. Future studies may show, however, that aerosolization of a surfactant preparation with optimal host defense properties may be feasible. In this situation, patients at risk of developing ARDS but with mild and uniform lung injury may benefit from the aerosolizaton technique. More research is required to support this approach, however.

\section{Novel indications for surfactant administration}

Recent studies have suggested that surfactant may be effective in patients with asthma because inhaled synthetic surfactant has been shown to abolish the early allergen-induced response in patients with asthma (10). Surfactant has also been shown to be effective in animal models and human trials of otitis media and sleep apnea, respectively $(11,12)$. However, these are preliminary studies and further research is required to verify its efficacy in these situations.

\section{CONCLUSIONS}

Exogenous surfactant administration may be effective for patients with established ARDS, but it would appear that treatment strategies need to be tailored to the specific patient involved. Ongoing and future studies will evaluate the role of surfactant alterations at very early stages of lung injury to determine whether prophylactic or early administration of surfactant can prevent the development of more severe disease.

\section{REFERENCES}

1. Possmayer F, Yu SH, Weber JM, Harding PG. Pulmonary surfactant. Can J Biochem Cell Biol 1984;62:1121-33.

2. Wright JR, Hawgood S. Pulmonary surfactant metabolism. Clin Chest Med 1989;10:83-93.

3. Veldhuizen RAW, Ito Y, Marcou J, Yao LJ, McCAig L, Lewis JF. Effects of lung injury on pulmonary surfactant aggregate conversion in vivo and in vitro. Am J Physiol 1997;16:L872-8.

4. Veldhuizen RAW, Marcou J, Yao LJ, McCaig L, Ito Y, Lewis JF. Alveolar surfactant aggregate conversion in ventilated normal and injured rabbits. Am J Physiol 1996;270:L152-8.

5. Pison U, Max M, Neuendank A, Weissbach S, Pietschmann S. Host defense capacities of pulmonary surfactant: Evidence of 'non-surfactant' functions of the surfactant system. Eur J Clin Invest 1994;24:586-99.

6. LeVine AM, Whitsett JA, Gwozdz JA, et al. Distinct effects of surfactant protein A or D deficiency during bacterial infection on the lung. J Immunol 2000;165:3934-40.

7. Lewis JF, Jobe AH. Surfactant and the adult respiratory distress syndrome. Am Rev Respir Dis 1993;147:218-33.

8. Zhu S, Ware LB, Geiser T, Matthay MA, Matalon S. Increased levels of nitrate and surfactant protein a nitration in the pulmonary edema fluid of patients with acute lung injury. Am J Respir Crit Care Med 2001;163:166-72.

9. Lewis JF, Veldhuzien R. The role of exogenous surfactant in the treatment of acute lung injury. Annu Rev Physiol 2003;65:613-42.

10. Babu KS, Woodcock DA, Smith SE, Staniforth JN, Holgate S, Conway JH. Inhaled synthetic surfactant abolishes the early allergen-induced response in asthma. Eur Respir J 2003;21:1046-9.

11. Feng LN, Chen WX, Cong R, Gou L. Therapeutic effects of eustachian tube surfactant in barotitis media in guinea pigs. Aviat Space Environ Med 2003;74:707-10.

12. Kirkness JP, Madronio M, Stavrinou R, Wheatley JR, Amis TC. Relationship between surface tension of upper airway lining liquid and upper airway collapsibility during sleep in obstructive sleep apnea hyperpnoea syndrome. J Appl Physiol 2003;95:1761-6. 


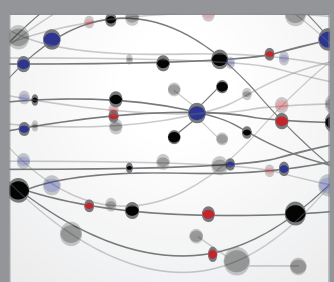

The Scientific World Journal
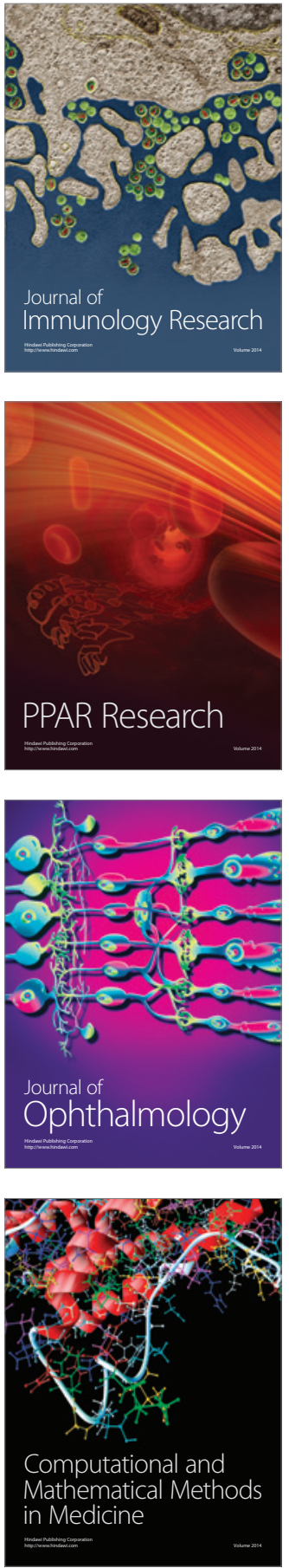

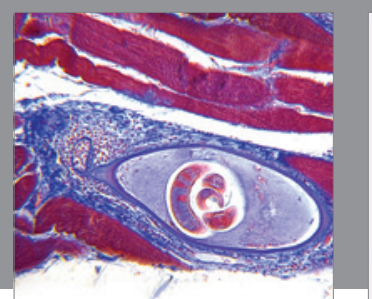

Gastroenterology Research and Practice

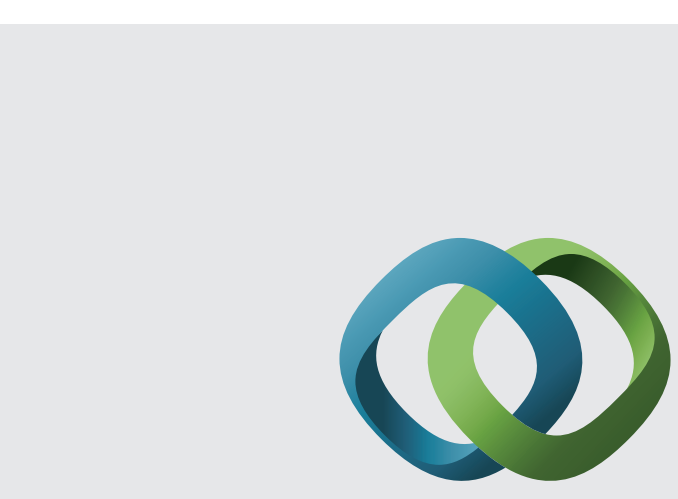

\section{Hindawi}

Submit your manuscripts at

http://www.hindawi.com
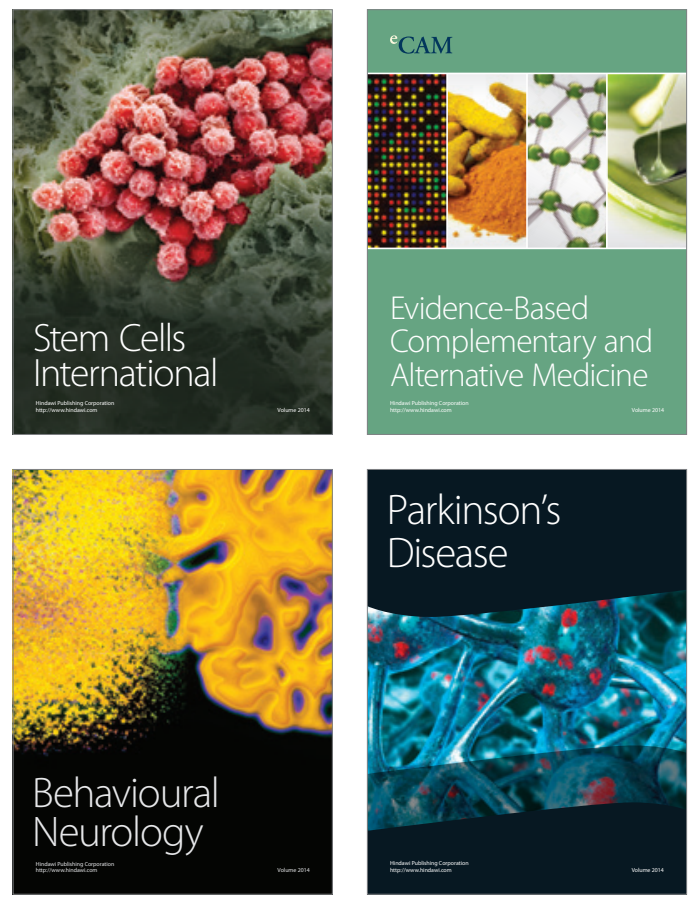
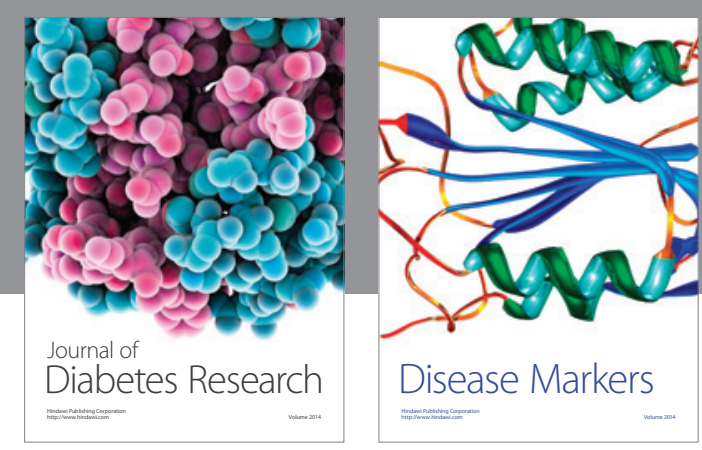

Disease Markers
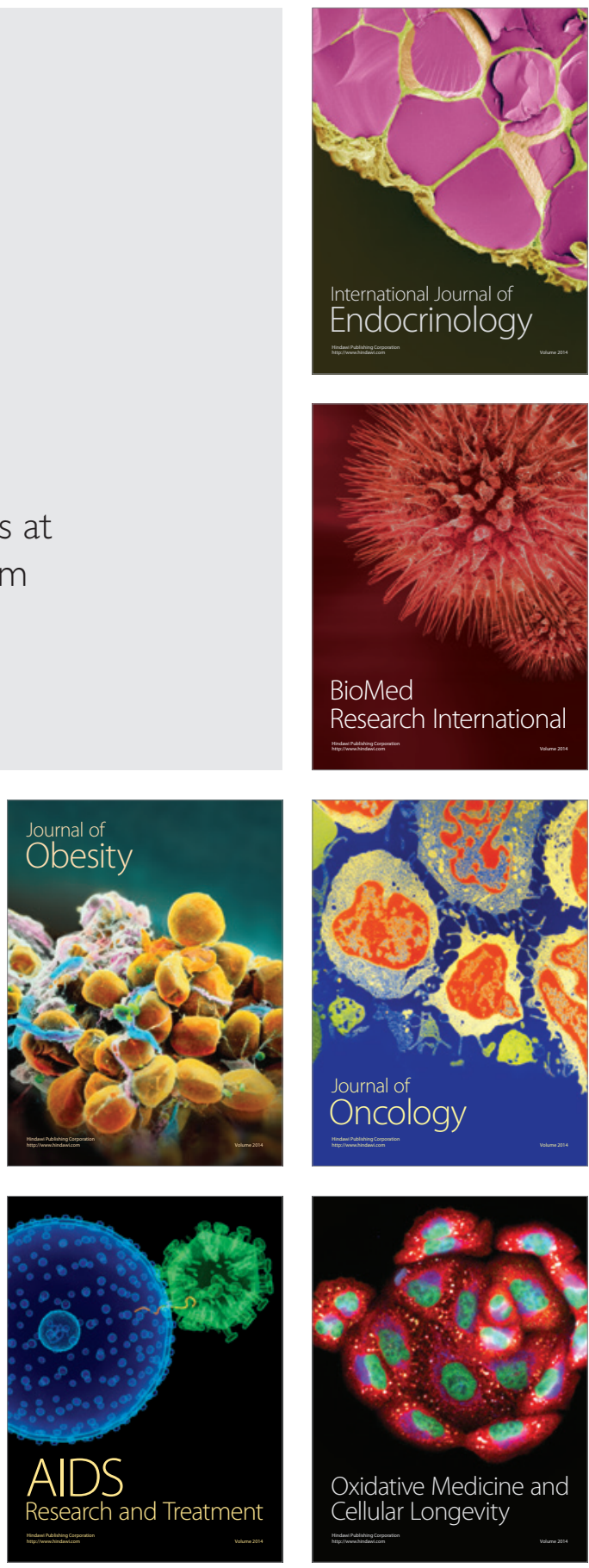